\title{
Total laparoscopic hysterectomy for non-puerperal uterine inversion: anatomical and operative considerations
}

\author{
Vasileios Minas $^{1} \cdot$ Antonios Anagnostopoulos $^{1} \cdot$ Nahid Gul $^{1}$
}

Received: 14 February 2015 / Accepted: 2 June 2015 /Published online: 2 July 2015

(C) Springer-Verlag Berlin Heidelberg 2015

\begin{abstract}
The diagnosis and management of non-puerperal uterine inversion can be challenging. The majority of cases are caused by benign leiomyomas, but $15 \%$ are related to a malignant mass. Published case reports can guide gynaecologists who encounter this rare condition and provide valuable insight in its management. We present a case of nonpuerperal uterine inversion in a pre-menopausal woman treated by total laparoscopic hysterectomy. We discuss the challenges we encountered due to the distorted pelvic anatomy and conclusions drawn from a literature review. The article is accompanied by relevant video material. A high level of suspicion is required for the diagnosis of non-puerperal uterine inversion. Morcellation techniques should be avoided due to the potential for malignancy. Where myomectomy is performed vaginally, the possibility of uterine rupture should be taken into account. Management by total laparoscopic hysterectomy has not been reported previously, but appears to be feasible. The technique should be meticulous and aim to identify by dissection important structures.
\end{abstract}

Keywords Total laparoscopic hysterectomy $\cdot$ Uterine inversion $\cdot$ Uterine myoma $\cdot$ Laparoscopy

Electronic supplementary material The online version of this article (doi:10.1007/s10397-015-0899-z) contains supplementary material, which is available to authorized users.

Vasileios Minas

billminas@gmail.com

1 Minimal Access Centre, Department of Obstetrics \& Gynaecology, Wirral University Teaching Hospital, Upton, UK

\section{Introduction}

Uterine inversion can occur either in the third stage of labour, when it is usually an iatrogenic complication due to premature traction of the umbilical cord, or in non-pregnant women as a result of a uterine mass which can be benign or malignant [1]. The majority of cases of non-puerperal uterine inversion occur in women over 45 years old. It is a rare clinical condition, and high level of suspicion is required to diagnose it [2]. The most common presenting symptom is abnormal vaginal bleeding. The patients may also complain of pelvic pain, presence of a mass in their vagina or protruding through the vagina, or acute urine retention. Although imaging can be of help, the diagnosis is often reached intra-operatively. Hysterectomy is recommended for women who have completed their families [2]. Here, we describe a case of a 47-year-old woman with nonpuerperal uterine inversion associated with a large uterine myoma managed by total laparoscopic hysterectomy.

\section{Case report}

A 47-year-old patient presented to her primary care physician with a recent onset history of heavy vaginal bleeding and symptoms of anaemia. Physical examination raised the suspicion of a vaginal/cervical mass, and she was referred to secondary care as an emergency. She was reviewed by the resident on-call gynaecologist in our unit the same day. Besides a raised body mass index (BMI of 35), her medical history was uncomplicated. Recent cervical cytology was normal. She was significantly anaemic and was transfused 3 units of packed red blood cells. Pelvic examination revealed a $6-8-\mathrm{cm}$ mass occupying the vagina in its entity. The mass was friable and bled on palpation. A fibroid protruding 
through the cervix was suspected; however, malignancy could not be excluded. An MRI was therefore arranged, and the patient was referred as an outpatient to a consultant gynaecologist with expertise in oncology and laparoscopic surgery on an urgent basis. The MRI suggested absence of pelvic lymphadenopathy and myometrial or parametrial invasion and supported the diagnosis of a fibroid. Fertility preservation was not an issue; therefore, the patient was consented for a hysterectomy.

On examination under general anaesthetic, vaginal access was limited due to the presence of the large fibroid. Its base appeared to be broad rather than pedunculated; therefore, a vaginal myomectomy was not attempted. Standard four-port laparoscopy was performed (video). It was not feasible to insert a uterine manipulator or a vaginal fornix delineator. Partial uterine inversion was diagnosed. The pelvic anatomy was distorted by the inward traction of the proximal part of the round and ovarian ligaments and fallopian tubes within the inverted uterine fundus. Bilateral salpingectomies were performed, and the ovaries were preserved. Bilateral ureterolysis was performed to identify the ureters which were seen drawn proximal to the uterus. Dissection of the ureters was continued down to the level of the uterine arteries which were clipped with Luger clips. Colpotomy was performed by digitally identifying the anterior vaginal vault above the invaginated fibroid. A total laparoscopic hysterectomy with bilateral salpingectomies and conservation of the ovaries was completed, and the specimen was extracted vaginally without the need for morcellation. The vaginal cuff was closed laparoscopically with four interrupted polyglactin absorbable sutures and extracorporeal knots. The estimated blood loss was less than $50 \mathrm{ml}$. The operating time was $146 \mathrm{~min}$. Recovery was uneventful, and histological examination proved a benign leiomyoma.

\section{Discussion}

Non-puerperal uterine inversion is usually caused by a benign leiomyoma. Malignancy accounts for $15 \%$ of the cases [2]. The diagnosis can be challenging and is often established intra-operatively. Gomez-Lobo et al. reported 150 cases of non-puerperal uterine inversions documented from 1887 to 2006 [3]. The majority have been managed by either vaginal or abdominal hysterectomy [3,4]. There is one case report which described a combined laparoscopic and vaginal approach to perform a total hysterectomy for a 40 -year-old patient with non-puerperal uterine inversion resulting from a 7$\mathrm{cm}$ leiomyoma attached to the uterine fundus [1]. To our knowledge, the case presented here is the first case of nonpuerperal uterine inversion managed by total laparoscopic hysterectomy.

Uterine preservation following extirpation of the mass has been described for women who wish to retain their fertility.
Rathod et al. adopted Kustner's method to manage a massive leiomyoma causing uterine inversion in a 28 -year-old woman [5]. Kustner's method involves anterior and posterior transection to the cervix by vaginal approach to replace the uterine fundus and has been used mainly in cases of peri-partum uterine inversion. The authors still had to perform a laparotomy to repair the uterine incision that occurred during vaginal myomectomy. De Vries et al. also encountered uterine rupture requiring laparotomy while performing a vaginal myomectomy for a pedunculated leiomyoma in a 19-year-old nulliparous woman with non-puerperal uterine inversion [6]. In another case, the Haultain's procedure was used successfully following vaginal myomectomy to replace the inverted uterus, by incising the cervical rim that entrapped the uterus [7].

In our case, we decided to avoid vaginal manipulation of the fibroid as we were concerned we might encounter significant haemorrhage due to its broad base. At laparoscopy, distortion of the pelvic anatomy required extensive ureterolysis and identification of the uterine arteries at the level where they cross the ureters to safely ligate them (video). Identification of the correct area to perform the colpotomy also proved difficult. Colpotomy was finally performed laparoscopically by inserting two fingers in the vagina above the fibroid and digitally palpating and applying tension onto the anterior vaginal vault. Indeed other authors have reported that the identification of the limit between the cervix and the vagina can be particularly laborious [8]. Auber et al. achieved complete devascularization of the uterus laparoscopically and thus identified the limit between the ischaemic reversed cervix and the normal vascularized vagina. They then used this limit to guide the colpotomy which they performed by vaginal route [1]. In retrospect, apart from the limited access, a vaginal approach to our case would place the ureters at risk due to the distorted pelvic anatomy; therefore, we recommend that the colpotomy can be performed laparoscopically with safety by the method described above.

\section{Conclusions}

In summary, non-puerperal uterine inversion is rare, and a high level of suspicion is required for diagnosis since both clinical examination and imaging can be misleading. Morcellation techniques should be avoided due to the potential for malignancy. Where myomectomy is performed vaginally, the possibility of uterine rupture should be taken into account. Management by total laparoscopic hysterectomy is feasible. The technique should be meticulous and aim to identify by dissection important structures, including the ureters and uterine arteries, as the anatomy can be distorted. We suggest digital palpation of the anterior vaginal fornix to guide the colpotomy. 
Compliance with ethical standards On behalf of all authors, the corresponding author states that there is no conflict of interest.

All procedures followed were in accordance with the ethical standards of the responsible committee on human experimentation (institutional and national) and with the Helsinki Declaration of 1975, as revised in 2008.

Informed consent was obtained from all patients for being included in the study.

Authors' contribution A Anagnostopoulos is responsible for data collection and manuscript writing. N Gul is responsible for the patient's clinical care. V Minas is responsible for video and manuscript writing/editing.

\section{References}

1. Auber M, Darwish B, Lefebure A, Ness J, Roman H (2011) Management of nonpuerperal uterine inversion using a combined laparoscopic and vaginal approach. Am J Obstet Gynecol 204:e7-e9
2. Lupovitch A (2005) Non-puerperal uterine inversion in association with uterine sarcoma: case report in a 26-year-old and review of the literature. Gynecol Oncol 97:938-941

3. Gomez-Lobo V, Burch W, Khanna PC (2007) Non-puerperal uterine inversion associated with an immature teratoma of the uterus in an adolescent. Obstet Gynecol 110:491-493

4. Lascaride E (1968) Surgical management of nonpuerperal inversion of the uterus. Obstet Gynecol 32:376-381

5. Rathod S, Samal SK, Pallavee P, Ghose S (2014) Non puerperal uterine inversion in a young female- a case report. J Clin Diagn Res 8:OD01-OD02

6. de Vries M, Perquin DA (2010) Non-puerperal uterine inversion due to submucous myoma in a young woman: a case report. J Med Case Rep 4:21

7. Tibrewal R, Goswami S, Chakravorty PS (2012) Non puerperal uterine inversion. J Obstet Gynaecol India 62:452453

8. Jones HW (1951) Non puerperal inversion of uterus. Am J Surg 81: 492-495 